\title{
Determinants of the Doppler flow velocity profile through the mitral valve of the human fetus
}

\author{
Ana-Maria Carceller-Blanchard, Jean-Claude Fouron
}

\begin{abstract}
Objective-A normal fetal flow velocity profile through the atrioventricular valves early in gestation is characterised by a higher late peak (A) than early peak (E) velocity waveform, whereas the E/A ratio is known to increase throughout pregnancy. This study aims firstly to identify which of the two variables, $E$ or $A$, is the contributory factor to the increased $E / A$ ratio and secondly to assess the relative influence of gestational age, heart rate, and stroke volume on the flow velocity profile through the fetal mitral valve.
\end{abstract}

Design-Eighty normal fetuses from 18 to 38 weeks of gestation were examined by Doppler echocardiography. The variables measured were $E$ and $A$ waves, the early and late flow velocity integrals (EI and $A I)$, and the total filling velocity integral (TI). The ratios E/A and EIAI were also calculated. Transvalvar flow was obtained by multiplying TI by mitral area. Associations between Doppler variables and gestational age, heart rate, and stroke volume were assessed by multifactorial Anova and simple or multiple stepwise regression analyses.

Results-The results showed that the heart rates found did not affect flow velocity variables. There were only weak correlations between both $A$ and $A I$ values and gestational age (negative) and volume load (positive). With the advance in gestation, a significant increase in the early filling $E$ wave was found. The $E$ wave was also positively correlated with stroke volume.

Conclusion-Contrary to the accepted concept that changes in fetal $E / A$ ratio are related to an improvement in ventricular compliance, this study shows that only the $E$ wave changes. Although these results cannot establish whether changes in the ventricular relaxation process or volume load are responsible for the progressive increase of the $E$ wave, indirect evidence suggests that ventricular relaxation is in fact the most important contributory factor.

(Br Heart f 1993;70:457-460)

Flow velocity waveforms through the atrioventricular (AV) valves during intrauterine life have been well documented. ${ }^{1-3}$ Contrary to what is normally found during extrauterine life, the fetal profile is characterised by a higher last peak (A) than early peak (E) velocity waveform. This waveform has been interpreted as an indication of a decrease in compliance of the fetal myocardium. With advancing gestational age, the fetal ratio E/A has been shown to increase progressively which has been interpreted as an improvement in myocardial compliance. ${ }^{2}$

Doppler derived indices of left ventricular function, however, are complex phenomena affected by the interaction of multiple factors. ${ }^{4-6}$ Whereas the $\mathrm{E}$ wave is related to active ventricular muscle relaxation, the $A$ wave is caused by atrial contraction and also influenced by ventricular compliance. The respective velocity profiles of the $E$ and $A$ waves are interrelated with each other. It is theoretically possible that during fetal life, an inadequate myocardial relaxation could impair early ventricular filling thereby allowing more blood to remain in the atrium for the last part of diastole, which would result in a higher A wave. This event would not necessarily be due primarily to a lack of myocardial compliance. Furthermore, in adult life other variables such as heart rate and loading conditions are known to influence the relation between the two ventricular diastolic filling waves independently of muscular diastolic function. Attention has not been given to the influence of these variables on fetal myocardial diastolic function.

It is the purpose of this study therefore, firstly to find which of the two elements of left ventricular filling dynamics, the $\mathrm{E}$ or $\mathrm{A}$ wave, is more affected during the progression of gestation, and secondly to evaluate systematically the interrelation between gestational age, heart rate, and stroke volume on Doppler flow velocities through the fetal mitral valve.

\section{Patients and methods}

Eighty fetuses with a gestational age ranging from 18 to 38 weeks were examined by Doppler echocardiography. Only one scan was performed on each infant. All fetuses had a normal morphological heart and were in sinus rhythm. Indications for echocardiographic scanning were either to rule out morphological anomalies of the heart or to investigate a possible arrhythmia, which turned out to be absent. Postnatal confirmation of normal cardiac findings have been sought in all infants. 
STUDY PROTOCOL

Real time and Doppler echocardiographic studies were performed either with an HP Sonos 500 model 77020 AC or Sonos 100 model $77570 \mathrm{~B}$ and a 5 or $7 \mathrm{MHz}$ transducer. Flow velocities through the AV valves were recorded in the four chamber view. To obtain maximal velocities through the mitral valve, the only results used in this study were from patients in whom it was possible to have the ultrasound beam parallel to, or at an angle of $<30^{\circ}$ to the presumed orientation of the blood flow. The sample volume was placed on or a few millimetres below the mitral valve annulus. Three maximum diameters of the valve were obtained by the same examiner and averaged. The mitral area was calculated with the formula: area $=\pi$ radius $^{2}$.

On each velocity curve, the following variables were measured or calculated: $E$ and $A$ waves, the $\mathrm{E} / \mathrm{A}$ ratio, $\mathrm{E}$ and $\mathrm{A}$ wave integrals (EI and $\mathrm{AI}$ ), the EI/AI ratio and the total filling velocity integral (TI). To overcome wave overlap, EI and AI were measured after drawing a line dividing the $\mathrm{E}$ and $\mathrm{A}$ waves vertically at their point of convergence. Transvalvar flow during each diastole was calculated by multiplying TI by mitral area which, in the absence of mitral regurgitation, was the equivalent of the left ventricular stroke volume. This value was used to assess the volume load, one aspect of the preloading condition of the fetal left ventricle throughout pregnancy. All values corresponded to the average of measurements taken on three consecutive complexes recorded at a speed of $100 \mathrm{~mm} / \mathrm{s}$.

\section{DATA ANALYSIS}

The mean values of the various Doppler variables were established in relation to gestational age by dividing the subjects into three groups: group 1,18 to 24 weeks of gestation ( $n=37)$; group 2,25 to 32 weeks $(n=26)$; and group 3,33 to 38 weeks $(n=17)$. ANOVA multifactorial analysis was used to compare the Doppler variables measured in each of these three groups. When a significant difference was found, $t$ tests were applied to find which of the groups were actually different. Simple regression analyses were also applied to assess possible correlations between Doppler variables, and gestational age, heart rate, and stroke volume. In the

Table 1 Mean echocardiographic data for the 80 patients

\begin{tabular}{lcccc}
\hline & $\begin{array}{l}\text { Group 1 } \\
(18-24 \text { weeks }) \\
(n=37)\end{array}$ & $\begin{array}{l}\text { Group 2 } \\
(25-32 \text { weeks }) \\
(n=26)\end{array}$ & $\begin{array}{l}\text { Group 3 } \\
(33-38 \text { weeks }) \\
(n=17)\end{array}$ & p value \\
\hline Indices & $23.67(5.04)$ & $27.05(6.26)$ & $30.67(6.44)$ & 0.01 \\
A (cm/s) & $40.38(9.45)$ & $39.27(10.39)$ & $36.33(7.03)$ & NS \\
E/A & $0.59(0.08)$ & $0.70(0.10)$ & $0.86(0.15)$ & 0.01 \\
EI (cm) & $1.33(0.53)$ & $1.63(0.42)$ & $1.94(0.6)$ & 0.01 \\
AI (cm) & $2.06(0.95)$ & $2.13(0.68)$ & $2.00(0.53)$ & NS \\
EI/AI & $0.66(0.13)$ & $0.80(0.18)$ & $0.98(0.22)$ & 0.01 \\
TI (cm) & $3.42(1.42)$ & $3.78(1.12)$ & $3.90(0.98)$ & NS \\
MA (cm $)$ & $0.37(0.11)$ & $0.61(0.14)$ & $0.86(0.26)$ & 0.01 \\
SV (ml) & $1.25(0.57)$ & $2.35(1.05)$ & $3.40(1.66)$ & 0.01 \\
HR (beats/min) & $146(11)$ & $139(10)$ & $136(9)$ & 0.01 \\
\hline
\end{tabular}

SV, stroke volume; HR, heart rate. Values are represented by means (SD); MA, mitral area; fo other abbreviations see text. event of a correlation with more than one factor, multiple stepwise regression analyses were also carried out to find which of these factors had a preponderant influence. Values are presented as the mean (SD).

\section{Results}

\section{DOPPLER MEASUREMENTS}

Table 1 shows the Doppler echocardiographic data of the 80 fetuses of the three gestational age groups, as well as the result of the ANOVA test comparing the three groups. A significant increase in peak and integral velocities of the $E$ wave was found as gestation progressed. The A wave, however, decreased slightly throughout gestation. These changes did not reached significance. The value for the E/A ratio started at about 0.5 and the EI/AI ratio at about 0.6 at mid-gestation and both increased to almost one by the end of gestation. Both increases were significant. The increase found in stroke volume throughout pregnancy for all three groups was significant between each group. Heart rate decreased slightly from an average of 146 beats $/ \mathrm{min}$ in group 1 to 139 beats $/ \mathrm{min}$ in group 2. There was no further change in heart rate between groups 2 and 3 .

\section{SIMPLE REGRESSION ANALYSIS \\ Effect of maturation}

Positive correlations were found between gestation and the $\mathrm{E}$ wave, $\mathrm{E} / \mathrm{A}$, and stroke volume (figure $1-\mathrm{A}, \mathrm{B}$, and $\mathrm{C}$ ). The EI/AI ratio also had a positive correlation with gestation $\left(\mathrm{EI} / \mathrm{AI}=0.02 \mathrm{x}+0.22 ; \mathrm{r}^{2}=0.36 ; \mathrm{p}<0.01\right)$. On the other hand, no significant correlation was noted between gestational age and the $A$ wave (figure D).

\section{Effect of heart rate}

There were negative correlations between heart rate and E/A $\left(\mathrm{p}<0.01 ; \mathrm{r}^{2}=0.15 ; \mathrm{E} / \mathrm{A}\right.$ $=-0.005 x+1.40)$, EI $\left(p<0.01 ; r^{2}=0.11 ;\right.$ $\mathrm{EI}=-0.02 \mathrm{x}+3.92), \mathrm{EI} / \mathrm{AI}$ ratio $(\mathrm{p}<0.01$; $\left.r^{2}=0.11 ; \mathrm{EI} / \mathrm{AI}=-0.006 \mathrm{x}+1 \cdot 69\right)$. No significant correlation was found between peak $\mathrm{E}$ or $\mathrm{A}$ velocities and heart rate.

\section{Effect of loading conditions}

All diastolic variables involving the $\mathrm{E}$ wave showed a positive correlation with the increase in stroke volume (table 2). A weak positive correlation was found for $\mathrm{AI}$ and EI/AI ratio.

\section{Multiple regression analysis}

Table 3 shows the stepwise regression analysis between the various diastolic indices measured and the three variables analysed (gestational age, heart rate, and stroke volume). Both $\mathrm{E}$ and $\mathrm{A}$ waves correlated positively with stroke volume. This effect was more pronounced for the $\mathrm{E}$ than the $\mathrm{A}$ wave. Whereas stroke volume was the only elemen that correlated significantly with the $\mathrm{E}$ wave throughout pregnancy, age of gestation had a further slight negative impact on the peak $A$ wave. Consequently, the E/A ratio was inde- 

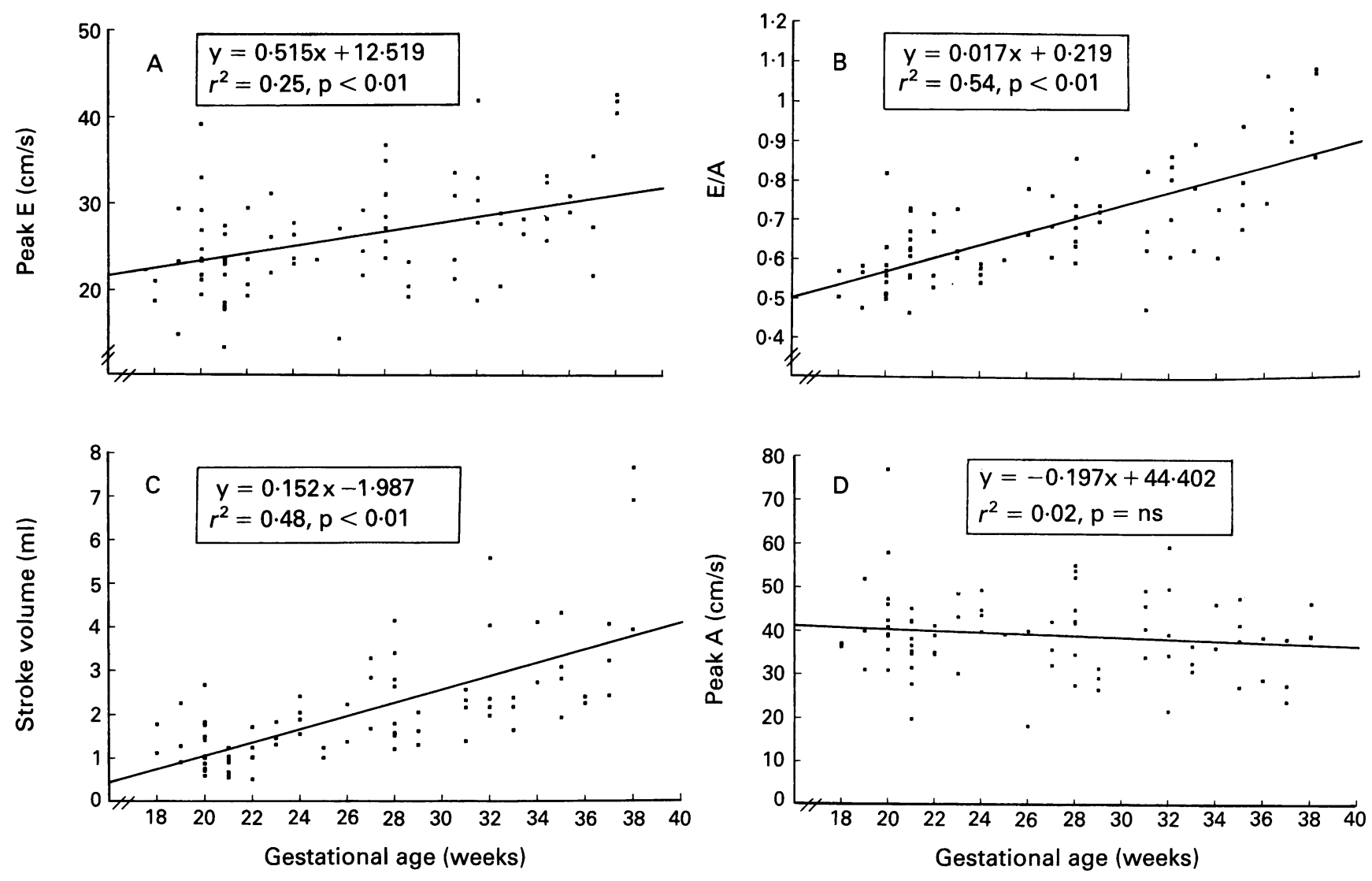

Correlations between gestational age and $(A)$ peak $E$ waves, $(B) E / A$ ratio, and $(C)$ stroke volume. No significant correlation was noted between gestational age and $A$ wave (D).

Table 2 Simple regression analysis between Doppler velocities and stroke volume

\begin{tabular}{llll}
\hline Indices & Pvalue & $r^{2}$ & Regression equation \\
\hline E $(\mathrm{cm} / \mathrm{s})$ & 0.01 & 0.55 & $\mathrm{E}=3.51 \mathrm{x}+19.03$ \\
$\mathrm{~A}(\mathrm{~cm} / \mathrm{s})$ & 0.02 & 0.07 & $\mathrm{~A}=1 \cdot 88 \mathrm{x}+35.29$ \\
$\mathrm{E} / \mathrm{A}$ & 0.01 & 0.30 & $\mathrm{E} / \mathrm{A}=0.06 \mathrm{x}+0.56$ \\
EI $(\mathrm{cm})$ & 0.01 & 0.51 & $\mathrm{EI}=0.30 \mathrm{x}+0.93$ \\
$\mathrm{AI}(\mathrm{cm})$ & 0.01 & 0.19 & $\mathrm{AI}=0.26 \mathrm{x}+1.54$ \\
EI/AI & 0.01 & 0.10 & $\mathrm{EI} / \mathrm{AI}=0.05 \mathrm{x}+0.67$ \\
\hline
\end{tabular}

pendent of stroke volume but increased progressively with advancing gestation. The velocity integrals of these respective indices followed a similar pattern with the exception of EI which, like stroke volume, was slightly affected by heart rate in a negative manner.

\section{Discussion}

The strict methodological requirements of an ultrasound beam parallel or less than $30^{\circ}$ from the direction of flow through the mitral valve was often difficult to obtain as the

Table 3 Stepwise regression analysis between Doppler velocities and age, heart rate and stroke volume

\begin{tabular}{lllll}
\hline Indices & Variables & $F$ & $r^{2}$ & Regression equation \\
\hline E & SV & 94.60 & 0.55 & $\mathrm{E}=3.51 \mathrm{SV}+19.03$ \\
$\mathrm{~A}$ & SV & 25.59 & 0.26 & $\mathrm{~A}=4.82 \mathrm{SV}-0.93 \mathrm{age}+53.92$ \\
E/A & Age & 19.81 & & \\
EI & SV & 92.37 & 0.54 & $\mathrm{E} / \mathrm{A}=0.02 \mathrm{age}+0.22$ \\
& HR & 73.73 & 0.54 & $\mathrm{EI}=0.29 \mathrm{SV}-0.01 \mathrm{HR}+2.34$ \\
AI & SV & 55.7 & & \\
EI/AI & Age & 22.24 & 0.37 & $\mathrm{AI}=0.5 \mathrm{SV}-0.08 \mathrm{age}+3.02$ \\
\hline
\end{tabular}

position of the fetus could not be controlled. This explains why only 80 fetuses out of a population of more than 1000 normal fetuses from our unit could fulfill the criteria. We also elected to place the pulsed Doppler sample at the level of, or a few millimetres below, the valve annulus because this ensured interrogation of flow at the same site for all fetuses, and also at the level where measurements of cross sectional area were made. Although this position could have some influence on the absolute values of $E$ and $A$ wave peak velocities, ${ }^{7}$ it is usually chosen in early life because of the small size of the heart.

In previous studies carried out on adults, transmitral flow velocity integral alone was used as a marker of ventricular preload. ${ }^{8}$ This was not possible in our group of fetuses as the valvar cross sectional area was increasing with gestational age. In fact, because of these increasing diameters, total integral velocities through the mitral valve did not change significantly with advancing gestation despite an increase of blood flow through the valve. The same reservation must be made for the interpretation of changes in AI and EI taken separately. Study of the evolution of their ratio, however, remains valid. Changes in heart rate were within the physiological range and had no significant effect on peak velocities of either diastolic wave.

Our study confirms previous findings that the $\mathrm{E} / \mathrm{A}$ ratio of the flow profile of the AV valves increases progressively with gestation. The data show however, that the $\mathrm{E}$ wave is 
mainly responsible for the change in the flow profile. The A wave shows little change throughout gestation. These findings do not support the accepted concept that the predominant $\mathrm{A}$ wave found during fetal life is essentially related to a lack of compliance of the ventricle. According to our data, the most important changes during gestation occur during the ventricular relaxation process. It is generally agreed that these flow velocity profiles are caused by changes in pressure gradients between the atria and ventricles during diastole. In early diastole, both the pressure within the atrium and the active process of myocardial relaxation are responsible for this gradient. Myocardial relaxation is sometimes referred to as the suction effect of the heart. An impairment in relaxation has been shown to correlate with a decrease in peak velocity of the $\mathrm{E}$ wave, ${ }^{5}$ as we found in our youngest group of fetuses. Our study further shows that the changes in peak $\mathrm{E}$ and EI correlate with changes in volume load. Experimental data and clinical studies on the adult myocardium have shown that the atrioventricular gradient can be modified significantly by a change in preload. ${ }^{89}$

Our results do not allow us to distinguish which of the two elements, preload or myocardial relaxation, is the primary event in the cause-effect relation. Some elements of our study, however, indirectly suggest that maturational changes in the intrinsic properties of the heart and not the loading condition, as partly reflected by the stroke volume, could be the primary event. In our group of fetuses, the stroke volume almost tripled from the middle to the end of gestation. If the intrinsic diastolic properties of the ventricle had not improved, a rise in venous pressure would have had to occur to explain the higher atrioventricular gradient early in diastole that led to a progressively higher $\mathrm{E}$ wave and to an increase in volume flow. As far as we know, a high fetal venous pressure has never been described at the end of gestation. Based on these findings, it would seem that the main reason for the tremendous increase in fetal cardiac output during gestation could actually be an improvement in active relaxation of the fetal myocardium; this could cause an increase in the early diastolic gradient that would lead to the progressively higher peak E velocity found with increasing gestation. This accords with the documented Doppler flow recordings of an increase in early diastolic forward flow in the inferior vena cava towards the end of gestation. ${ }^{10}$ Furthermore, the finding by Areias and coworkers of a significant increase in $\mathrm{E}$ amplitude without changes in $\mathrm{A}$ during the first weeks after birth would indicate that the intrauterine improvement in ventricular relaxation reported in the present study extends into early extrauterine life. ${ }^{11}$

A comprehensive view of the fetal left ventricular filling pattern can therefore be proposed as follows: early in gestation poor ventricular relaxation results in a small atrioventricular gradient and consequently, less blood enters the left ventricle in this early part of diastole. A greater volume of blood remains in the atrium at the time of atrial contraction, causing a higher AV gradient and a higher peak A velocity. Fetal maturation is associated with an improvement of ventricular relaxation causing progressively higher peak $\mathrm{E}$ waves. A progressively greater amount of blood flows into the ventricle during the early part of diastole causing a progressive increase of $\mathrm{EI}$ and the EI/AI ratio, approaching the pattern known to be present during extrauterine life. Clinical assessment of the function of ventricular diastole in the fetus should take into consideration these fundamental maturational changes.

We thank Susan Pamela Drblik for her advice and Elise Manseau and Nathalie Ishmael for their secretarial work.

1 Hata T, Hata K, Takamiya O, Kitao M. Fetal ventricular relaxation assessed by Doppler echocardiography. fournal of Cardiovascular Ultrasonography 1988;7: 207-13.

2 Reed KL, Sahn DJ, Scagnelli S, Anderson CF, Shenker L. Doppler echocardiographic studies of diastolic function in the human fetal heart: changes during gestation. f Am Coll Cardiol 1986;8:391-5.

3 Rizzo G, Arduini D, Romanini C, Mancuso S. Dopple echocardiographic assessment of atrioventricular velocity waveforms in normal and small-for-gestational-age fetuses. Br 7 Obstet Gynaecol 1988;95:65-9.

4 Devereux RB. Left ventricular diastolic dysfunction: early diastolic relaxation and late diastolic compliance. $\mathcal{F ~} \mathrm{Am}$ diastolic relaxation and late
Coll Cardiol 1989;13:337-9.

5 Thomas JD, Weyman AE. Echocardiographic Dopple evaluation of left ventricular diastolic function: physics evaluation of left ventricular diastolic function
and physiology. Circulation 1991;84:977-90.

6 Zoghbi WA, Bolli R. The increasing complexity of assessing diastolic function from ventricular filling dynamics. f Am Coll Cardiol 1991;17:237-8.

7 Johnson GL, Moffett CG, Jurnalov CD, Noonan JA. Effect of sample volume location on pulsed Doppler diastolic flow parameters in newborn infants. Pediat Cardiol 1988;9:221-4.

8 Berk MR, Gongyuan X, Kwan OL, Knapp C, Evans J, Kotchen $\mathrm{T}$, et al. Reduction of left ventricular preload by lower body negative pressure alters Doppler transmitral filling patterns. $\mathcal{F} \mathrm{Am}$ Coll Cardiol 1990;16: mitral fillin $1387-92$.

9 Coutois M, Vered Z, Barzilai B, Ricciotti N, Pérez JE, Ludbrook PA. The transmitral pressure-flow velocity Ludbrook PA. The transmitral pressure-flow velocity relation: effect of

10 Reed KL, Appleton CP, Anderson CF, Shenker L, Sahn DJ. Doppler studies of vena cava flows in human fetuses, insights into normal and abnormal cardiac physiology. Circulation 1990;81:498-505.

11 Areias JC, Meyer R, Scott WA, Goldberg SJ. Serial echocardiographic and Doppler evaluation of left ventricular diastolic filling in full-term neonates. $A m \mathcal{F}$ Cardiol 1990;66:108-11. 